\title{
Mobile Based Electricity Bill Deposit System through UML
}

\author{
Pawan Kumar Chaurasia ${ }^{1}$, Vipin Saxena ${ }^{2}$ \\ ${ }^{1}$ Department of Information Technology, Babasaheb Bhimrao Ambedkar University, Lucknow (U.P), India; ${ }^{2}$ Department of Compu- \\ ter Science, Babasaheb Bhimrao Ambedkar University, Lucknow, India. \\ Email: pkc.gkp@gamil.com, vsax1@rediffmail.com
}

Received January $5^{\text {th }}$, 2011; revised March $10^{\text {th }}, 2011$; accepted March $12^{\text {th }}, 2011$.

\begin{abstract}
The present paper deals with a model design through Unified Modeling Language (UML) for a mobile based electricity bill deposit system. Due to complex life style of people this model is proposed in the form of UML Class, Sequence and Use Case diagrams. For implementation of proposed model, a real case study of Uttar Pradesh Electricity Bill deposit System is considered. By the use of this model, one can display the status of deposited electricity bill on a hand held mobile device system.
\end{abstract}

Keywords: UML, Mobile Device, Electricity Bill, UML Class, UML Sequence \& UML Use Cases

\section{Introduction}

UML is a unique and most popular modeling language which has been used by various researchers for producing the object oriented designs. Slowly-slowly Software Industries are shifting their old structured design models in the form of object oriented design models by the use of UML. But, researchers are not emphasizing towards the use of UML for creating the Object Oriented Databases which are a fundamental requirements of the Object Oriented Models. Therefore, the present paper is an attempt to create Object Oriented Database through UML.

Booch $[1,2]$ has introduced the notations of UML which are used for the Object-Oriented analysis and Design with applications. For reducing the complexity of the structured designs, the three researchers namely G. Booch, James Rambugh and Ivar Jacobson [3-6] have introduced the object orientation in the software designs as well as in the software higher level languages which are nowadays most popular among the Software Industries/Software Professionals/Researchers. This representation is called as a meta-model, which describes the objects, attributes and relationship necessary to represent the concepts of UML within a software application. Malik, Dragos and Johan [7] presented a model-based testing approach for integration UML, UML-B and the Qtronic test generator tool for increasing the quality of models through test case generations. UML is easily translated into real world applications. Using this modeling language one can develop the software in a valuable and resourceful manner $[8,9]$.

UML class diagrams are the most important structural model and indeed the central model for researchers and developers. Unified Modeling Language is a widespread visual language provided by Object Management Group (OMG) for the construct, description, documents and modeling of systems software and processes. The initial version of UML (UML 1) originated with three leading object-oriented methods (BOOCH, OMT and OOSE). One of the purposes of UML is to provide the development community with a stable and common design language i.e. it is programming language independent. UML diagrams are grouped into language units. It describes Semantics, Notations and Standard Profiles. UML can define the model specification into structure, behavior and concepts [10].

UML 2.0 Superstructure models are serialized in XML 2.1 \& XML files containing package merges are semantically equivalent to the same XML files with the package merges merged away [11]. Object Management group (OMG) promotes the theory and practice of object-oriented technology in software development [12]. Smith initiated the software performance engineering and summarizes the UML. Pooley et al. [13-14] described the software process and lifecycle and explained how object oriented and component based engineering affect the de- 
sign process.

In the present work, object oriented database has been created for a real case study of Uttar Pradesh Electricity Bill Deposit System. Recently Government of Madhya Pradesh started the online electricity billing through Internet using cash card [15]. In the current Electricity Bill Deposit System, customer has to go to the Bill Section of the Electricity Department and can pay through cash or cheque. But by the use of proposed model, the Customer can pay the amount of Electricity Bill through a hand held Mobile Device by entering the credit/debit card number and within a few seconds, customer will get a SMS of the paid amount. In the proposed model, a UML Class, Sequence and UML Use Case diagrams are given.

\section{Uml Modeling for Electricity Bill Deposit System}

\subsection{UML Class Diagram}

The static representation of the software problems can be explained by the of the UML Class diagram. The purpose of a class diagram is to depict the classes within a model. In an object oriented application, classes have attributes, operations and relationships with other classes. The present work emphasized on the object oriented databases as shown in the following Figure 1. In this diagram, a customer (CUSTOMER Class) has a mobile device (MOBILE_DEVICE Class) and card (CARD Class) which may be credited (CREDIT_CARD Class) or debit (DEBIT_

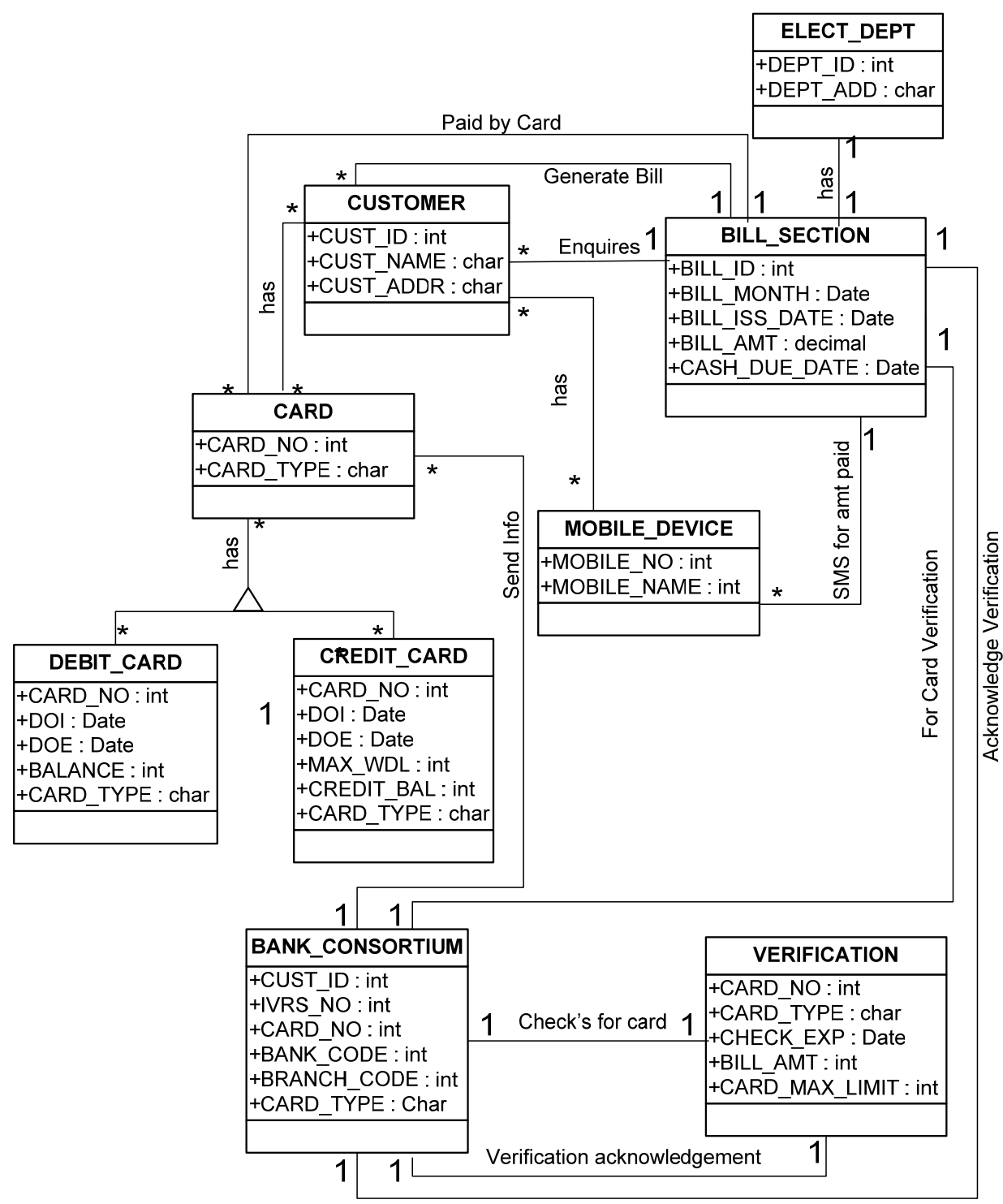

Figure 1. UML class diagram for mobile based electricity bill deposit system. 
CARD Class) card. The customer enquires from the billsection (BILL_SECTION Class) through network available on the mobile device. The main role of this bill section is to generate the bills and after the payment of the bills, this section sends a copy to the Electricity Department (ELECT_DEPT Class) and SMS to the mobile device of the customer. The payment of the bill is only done after the verification of the customer card by the bank consortium (BANK_CONSORTIUM Class) which has separate verification department (VERIFICATION Class).

\subsection{UML Sequence Diagram}

A sequence diagram has two dimensions. The vertical dimension shows the sequence of messages/calls in the time order that they occur, the horizontal dimensions shows the object instances to which the messages are sent. Sequence diagram shows the relationship between classes arranged in a time sequence. In the given sequence diagram as shown in Figure 2, there are three objects. Customer (CUSTOMER Object) sends enquiries for a bill, bill section (BILL_SECTION Object) of the electricity department generates a bill. Customer paid a bill by card using credit/debit card. The bill section sends a card for verification into a bank. Then the verification section of the bank (BANK_CONSORTIUM Object) verifies the card and acknowledges to the bill section of the electricity department. Bill section sends SMS for amount paid to the customer.

\subsection{UML Use Case Diagram}

A use case diagram is a diagram which shows a set of use cases and actors and their relationships. Use case diagrams commonly contain the use cases, the actors, the generalization relationship between actors, the precede relationships between use cases and the association relationships between actor and use cases. Model captures the important aspects of the thing being modeled from a certain point of view and simplifies or omits the rest. Model of a software system is made in a modeling language such as UML. The model has both semantic and can take various forms that include both pictures and text. A use case diagram is shown below in Figure 3.

\section{Concluding Remarks}

From the above work it is concluded Mobile Based UML Modeling for Electricity Bill Deposit System is only effective in those areas where the computerization has been done. By the proposed UML model, CUSTOMER enquires the bill from the BILL_SECTION and paid through the CARD. Bank verifies the payment and sends the acknowledgement to the BILL_SECTION. BILL_

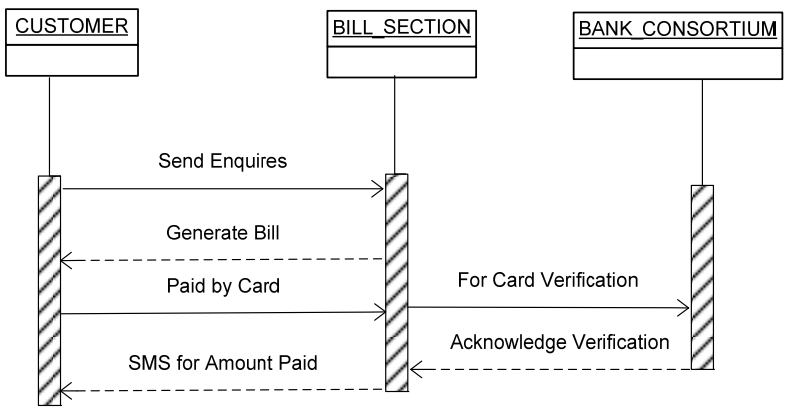

Figure 2. UML sequence diagram for mobile based electricity bill deposit system.

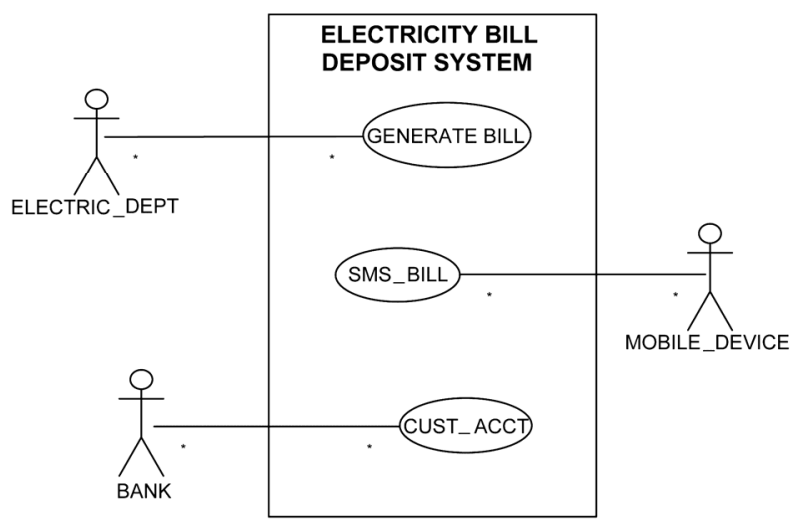

Figure 3. UML use case diagram for mobile based electricity bill deposit system.

SECTION sends SMS on the customer hand held device MOBILE_DEVICE. In the above model, object oriented database has been designed and a large database can be stored by the use of data cube methods. The above work can also be extended for mining the data from cubes and one can get the desired information within a fraction of seconds on hand held device.

\section{Acknowledgments}

The authors are very thankful to Prof. B. Hanumaiah, Vice-Chancellor, Babasaheb Bhimrao Ambedkar University (A Central University), Vidya Vihar, Rae Bareli Road, Lucknow, India for providing excellent computation facilities in the University campus. Thanks are also due to University Grant Commission, New Delhi for providing research funding to the University.

\section{REFERENCES}

[1] G. Booch and B. Cummings, “Object Oriented Analysis and Design,” Addison Wesley, Menlo Park, 1994.

[2] G. Booch, "Growing the UML Software and System Modeling,” Vol. 1, No. 2, December 2002, pp. 157-160. doi:10.1007/s10270-002-0013-7 
[3] G. Booch, "Object Solutions: Managing the Object Oriented Project,” Addison Wesley, Menlo Park, 1996.

[4] G. Booch, J. Rumbaugh and I. Jacobson, "The Unified Modelling Language User Guide,” Addison-Wesley, Reading, 1999.

[5] J. Rumbaugh, M. Blaha, W. Lorensen and F. Eddy, "Object Oriented Modeling and Design,” Prentice Hall, Englewood Cliffs, 1991.

[6] I. Jacobson "Object Oriented Software Engineering: A Use Case Driven Approach”, Addison Wesley, New York, 1992.

[7] Q. A. Malik, D. Truscan and J. Lilius, "Using UML Models and Formal Verification in Model-Based Testing," 17th IEEE International Conference and Workshops on the Engineering of Computer-Based Systems, Oxford, 22-26 March 2010, pp. 50-56.

[8] S. K. Mostéfaoui, "A Context Model Based on UML and XML Schema Representations," Proceedings of the IEEE/ACS International Conference on Computer Systems and Applications, 31 March-4 April 2008, Doha, pp. 810-814.
[9] J. Rumbaugh, I. Jacobson and G. Booch, “The Unified Modeling Language Reference Manual,” Addison-Wesley, Reading, 1999.

[10] OMG “Unified Modeling Language Specification,” 2010. http://www.omg.org.

[11] OMG, "UML Superstructure Specification, V2.0,” 2004. http://www.omg.org/cgi-bindoc?formal/05- 07-04.

[12] OMG, "Unified Modeling Language Specification, Version 1.3,” June 1999. http://www.rational.com/media/uml/post.pdf

[13] R. Pooley and P. King, "The Unified Modeling and Performance Engineering," Proceedings of IEEE Software, Vol. 146, No. 1, February 1999, pp. 2-10. doi:10.1049/ip-sen:19990151

[14] R. Pooley and P. Stevens, "Using UML: Software Engineering with Object and Components,” Addison-Wesley Longman, California, 2000.

[15] "How to Pay Electricity Bill through MPOnline," 30 September 2010. http://www.mponline.gov.in 